\title{
Monochromator for Aberration-Corrected STEM
}

Masaki Mukai ${ }^{1}$, Eiji Okunishi ${ }^{1}$, Masanori Ashino ${ }^{1}$, Kazuya Omoto $^{1}$, Tomohisa Fukuda ${ }^{1}$, Akihiro Ikeda ${ }^{1}$, Kazunori Somehara ${ }^{1}$, Toshikatsu Kaneyama ${ }^{1}$, Tomohiro Saitoh ${ }^{2}$, Tsukasa Hirayama ${ }^{2}$, Yuichi Ikuhara ${ }^{2,3}$

1. JEOL Ltd., 3-1-2 Musashino, Akishima, Tokyo 196-8558, Japan

2. Japan Fine Ceramics Center, 2-4-1 Mutsuno, Atsuta-ku, Nagoya, 456-8587, Japan

3. The University of Tokyo, 2-11-16 Yayoi, Bunkyo-ku, Tokyo 113-8656 Japan.

In a scanning transmission electron microscope (STEM), the elemental analysis at atomic resolution is realized by the combination with electron energy-loss spectroscopy (EELS), since an aberration corrected probe forming lens system enables us to obtain an electron probe sized about $0.1 \mathrm{~nm}$ [1]. In EELS, the energy resolution is mainly limited by the energy spread of the electron source, which is approximately $0.7 \sim 1.0 \mathrm{eV}$ for a Schottky source and $0.3 \sim 0.5 \mathrm{eV}$ for a cold field emission source. For the analysis of a detailed electronic state at an atomic scale at higher energy resolution, we developed a monochromator for an aberration-corrected STEM, which integrates a double Wien-filter system. [2]

The developed monochromator, which is placed between an extraction anode of Schottky source and an accelerator tube, consists of two Wien-filters and an energy selection slit inserted between the two filters. Figure 1 shows the results of the numerical calculation of the electron trajectories from the electron source to the plane of the exit crossover of the monochromator. The electron trajectories inside of the monochromator are set to be symmetric to the slit plane so that the energy-dispersion at the slit plane is cancelled at an exit plane. Therefore, the electron probe at the specimen plane is achromatic and stigmatic. The obtained energy-spread of the electron probe is controllable independently with the probe size by choosing the width of the slit. In addition, the accelerating voltage is changeable independently on the setting of the monochromator and the electron trajectories inside the monochromator, since the accelerator of the electron gun is located after the monochromator and the potential along the optical axis inside the monochromator is kept constant. The ultimate energy resolutions of $36 \mathrm{meV}$ and $30 \mathrm{meV}$ were obtained with $0.1 \mathrm{~s}$ acquisition and the slit of $0.25 \mu \mathrm{m}$ at $200 \mathrm{kV}$ and $60 \mathrm{kV}$, respectively.

Figures 2 show carbon K-edge spectra from a diamond, as an example showing the difference in the energy-loss near-edge structure (ELNES) on the different energy resolutions. Figure 2 (a) was obtained with a Schottky source and its energy resolution is approximately $1 \mathrm{eV}$. The spectra obtained with the slits of $4 \mu \mathrm{m}, 2 \mu \mathrm{m}$ and $0.5 \mu \mathrm{m}$ at $200 \mathrm{kV}$ are shown in Figs. 2 (b)-(d), respectively. With the monochromated electron source, a core exciton peak, which was unable to be detected with a standard schottky, is clearly detectable. And a dip, appeared between the main peak of K-edge and the exciton peak approximately at $289 \mathrm{eV}$, was getting deeper as the energy resolution is getting higher. In order to investigate the analytical performances with both of high energy resolution and high spatial resolution, we tried the atomic resolution EELS mapping using a $\mathrm{SrTiO}_{3}$ specimen along the $<100>$ direction obtained with monochromated electrons whose energy spread is $142 \mathrm{meV}$ at $60 \mathrm{kV}$, as shown in Fig. 3. Thus, the atomic columns of $\mathrm{Sr}$ and $\mathrm{Ti}$ in a $\mathrm{SrTiO}_{3}$ have been successfully visualized using the monochromated electrons with $142 \mathrm{meV}$ energy spread.

\section{References:}

[1] E. Okunishi et al.: Microscopy \& Microanalysis, vol.13 (supple.2), 1150-1151 (2006)

[2] M. Mukai et al.: Microscopy \& Microanalysis, vol.19 (supple.2), 1126-1127 (2013) 


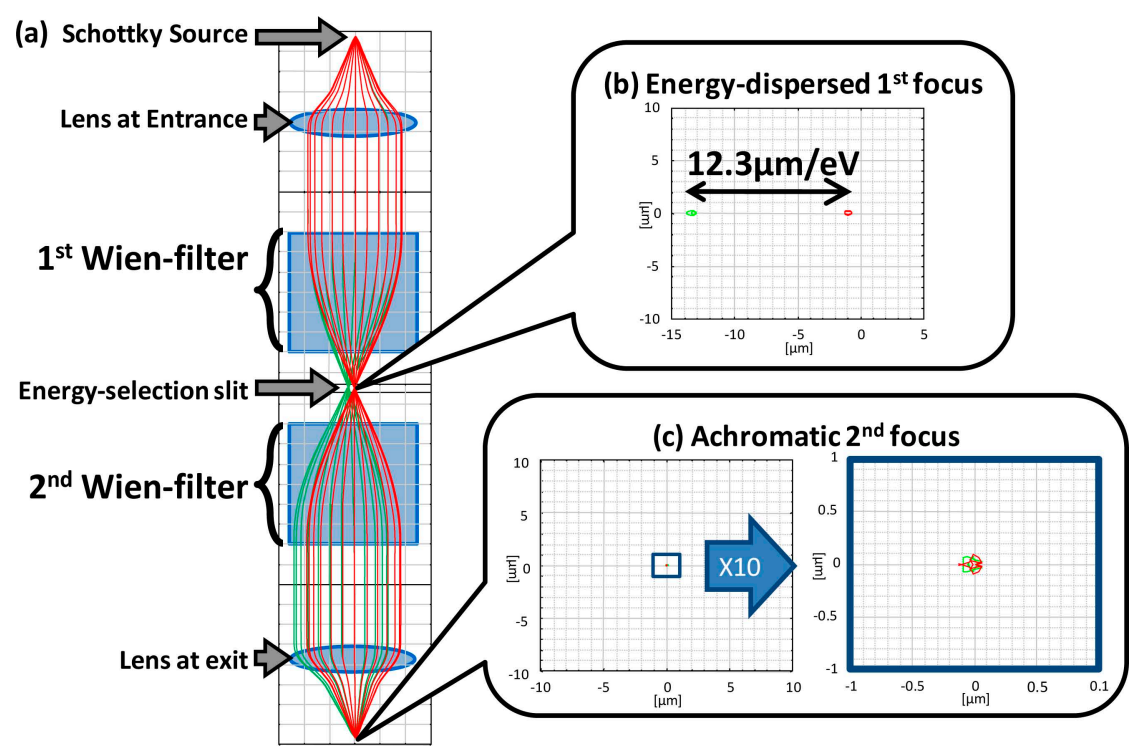

Fig. 1. (a) Calculated trajectories along optical axis from source to exit of monochromator, (b) beam shapes at slit plane with an energy-dispersed 1st focus and (c) beam shapes at exit plane with an achromatic $2^{\text {nd }}$ focus. The red lines and the green lines show the trajectories having energies of 1400 and $1401 \mathrm{eV}$ inside of the monochromator.

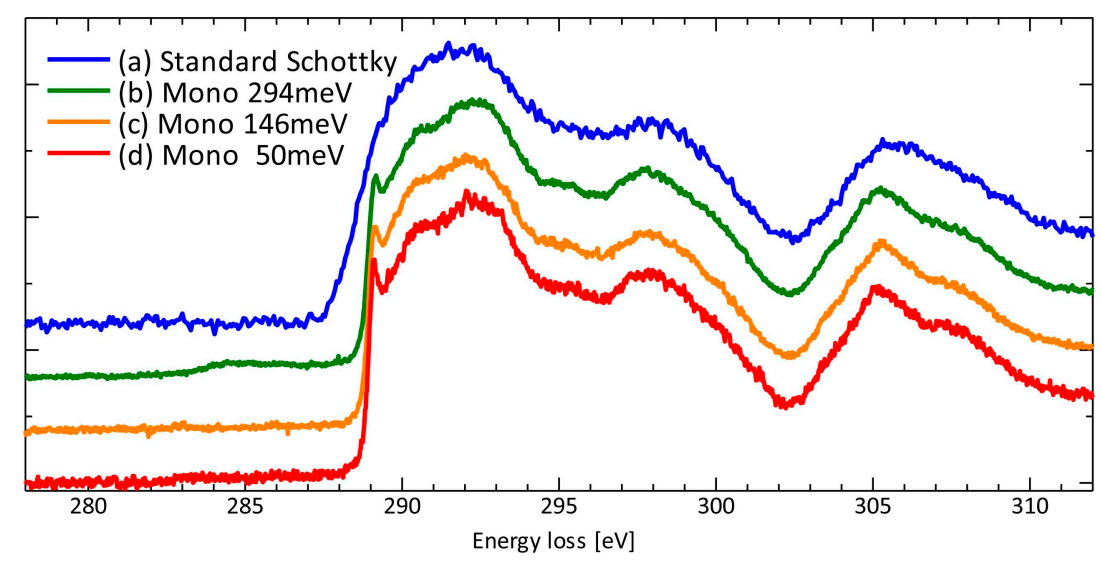

Fig. 2. Raw spectra of carbon K-edge of diamond obtained at $200 \mathrm{kV}$, using (a) Schottky source, (b)-(d) monochromated sources with energy spreads of $294 \mathrm{meV}, 146 \mathrm{meV}$ and $50 \mathrm{meV}$, respectively.

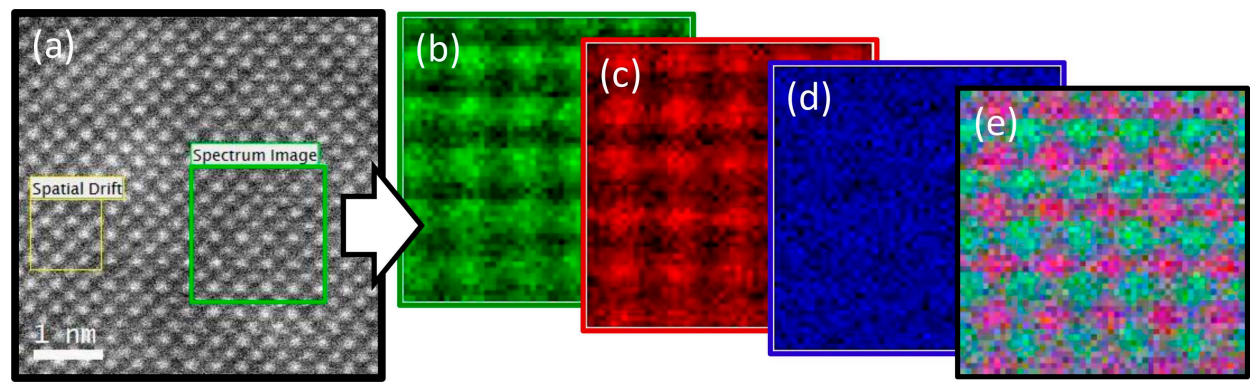

Fig. 3. Atomic resolution EELS map of $\mathrm{SrTiO} 3$ [100] recorded at $60 \mathrm{kV}$ with monochromated electrons of $142 \mathrm{meV}$ energy spread. The probe current was $19 \mathrm{pA}$ and dwell time in each pixel was $0.01 \mathrm{sec}$., (a) HAADF, (b) Sr M-map, (c) Ti L-map, (d) O K-map, (e) Overlay map. 